\title{
Dealing with uncertainties in fusion power plant conceptual development
}

\author{
R. Kemp ${ }^{1,2}$, H. Lux ${ }^{1}$, M. Kovari ${ }^{1}$, J. Morris ${ }^{1}$, R. Wenninger ${ }^{3,4}$, H. Zohm ${ }^{4}$, W. Biel ${ }^{5}$ and \\ G. Federici ${ }^{3}$ \\ ${ }^{1}$ CCFE, Culham Science Centre, Oxfordshire OX14 3DB, United Kingdom \\ ${ }^{2}$ EUROfusion PMU, Culham Science Centre, Oxfordshire OX14 3DB, United Kingdom \\ ${ }^{3}$ EUROfusion PMU, Boltzmannstr.2, Garching 85748, Germany \\ ${ }^{4}$ IPP, Boltzmannstr.2, Garching 85748, Germany \\ ${ }^{5}$ FZ Jülich, Jülich 52425, Germany
}

Corresponding Author: richard.kemp@ukaea.uk

\begin{abstract}
:
Although the ultimate goal of most current fusion research is to build an economically attractive power plant, the present status of physics and technology does not provide the performance necessary to achieve this goal. Therefore, in order to model how such plants may operate and what their output might be, extrapolations must be made from existing experimental data and technology. However, the expected performance of a plant built to the operating point specifications can only ever be a "best guess". Extrapolations far beyond the current operating regimes are necessarily uncertain, and some important interactions, for example the coupling of conducted power from the scape-off layer to the divertor surface, lack reliable predictive models. This means both that the demands on plant systems at the target operating point can vary significantly from the nominal value, and that the overall plant performance may potentially fall short of design targets.

In this contribution we discuss tools and techniques that have been developed to assess the robustness of the operating points for the EU-DEMO tokamak-based demonstration power plant, and the consequences for its design. The aim is to make explicit the design choices and areas where improved modelling and DEMO-relevant experiments will have the greatest impact on confidence in a successful DEMO design.
\end{abstract}

\section{Introduction}

The ultimate goal of research into fusion for energy is to supply electricity economically, sustainably, and safely. At some point we must demonstrate that fusion is a credible energy source. The target of the EU DEMO strategy is that DEMO should demonstrate significant net electrical power for significant time; tritium self-sufficiency; and achieve a functional lifetime demonstration of all relevant supporting technology including divertor, remote handling systems, etc. [1]. A target of $500 \mathrm{MW}$ net electrical power and pulse length of $2 \mathrm{hrs}$ are considered sufficient to provide a margin to accomplish these goals 
allowing for the inevitable variation between initial design and final operation; while these targets would not be attractive for a commercial reactor, DEMO is intended to demonstrate the integrated operation of power plant technologies rather than compete in the energy markets. These choices are explored more fully in reference [2]. The DEMO design point will build on ITER, which should demonstrate robust burning plasma physics regimes using a conventional divertor, and the validity of breeding blanket technologies. The assumption that ITER will be successful in demonstrating controlled burning plasma physics allows the conceptual design and evaluation of an "early DEMO" with wellestablished technology and regimes of operation.

However, there are significant technical hurdles to overcome before DEMO can be built and electricity generated from fusion can be commercialised. In particular, a fusion power plant capable of supplying electricity reliably and economically is far beyond the scale of existing experimental machines, including ITER. Furthermore, there is a shortage of suitable facilities for testing components in simulated fusion conditions. To design a demonstration plant such as DEMO, we must extrapolate from current physics and engineering knowledge into areas where we cannot be confident that performance will be as we expect at the design stage.

There are three approaches that can be taken to reducing this risk:

1. build in performance margins so that the nominal design targets are sufficiently in excess of the minimum acceptable performance;

2. perform research and development to reduce uncertainties in extrapolation;

3. reduce the minimum acceptable performance so that success can be claimed whatever is achieved.

Option 3 is clearly unacceptable in a major publicly-funded research programme; options 1 and 2 are complementary: as uncertainties in performance are reduced, performance margins can be narrowed, reducing the costs of the device in question. However in order to judge the appropriate margins and target research and development at the most effective areas, it is important to understand which physics and technology uncertainties have the largest effects on overall plant performance.

How these uncertainties propagate through plant design can depend on the integration between plant systems, and it may be that particular design choices can mitigate the effects of uncertainty - that is, the optimal design for which expected performance is robust against uncertainties may well not be the same as the design point found through optimisation based on a single set of assumed parameters. The interaction between these effects must be consistently captured and many design options explored.

System codes representing a fusion power plant capture the interactions between (usually relatively simple) models of all the important plant subsystems and are used to identify design points based on assumptions about the plasma performance and technology. Given the very large potential number of such design points, a single point for detailed assessment can be chosen by optimising a figure of merit such as major radius or pulse length. The systems code PROCESS [3, 4] has been used for past studies such as the 
European Power Plant Conceptual Study [5], and is now being used for the development of baseline designs to underpin EU DEMO design studies [2]. The purpose of using the systems code is to rapidly identify potential solution spaces without having to carry out complex analysis at every point. PROCESS is under continuous development to improve models and incorporate new data. The physics basis of DEMO is also under development to identify and investigate areas of significant uncertainty [6].

In order to proceed with detailed conceptual development across the full range of (highly-integrated) DEMO systems, a relatively stable baseline design point is essential. This design point, based on well-assessed physics and technology models, provides the basic performance requirements and expectations from each system [7]. Although this baseline design is the focus for much of the EUROfusion conceptual development work, a range of alternative concepts is also being explored and assessed with the aim of ensuring the final architecture chosen not only maximises the chance of success of the DEMO programme, but also fully supports the long-term goals of commercial fusion power. The EU DEMO approach is a systems engineering approach, and how uncertainties can be classified and incorporated into the design, and alternative architectures can be used to mitigate high-level uncertainties are discussed elsewhere [8, 9].

\section{Propagating uncertainties through DEMO design}

The EUROfusion Roadmap is currently being redrafted to take account of ITER delays [10] and advantage is being taken of this to expand the range of possible basic DEMO design points. As evaluation of these design points proceeds, less attractive and less achievable designs can be removed from the process (figure 1). Part of the evaluation must include not just the nominal performance and technical targets but also the robustness of the design against underperformance. For this a framework must be developed which systematically assesses the expected range of performance of a given design point, taking into account uncertainties in physics performance and technological abilities.

The approach to assessing the expected performance of a DEMO design is to attempt to answer the question: if we build it, how is it likely to behave? We begin by fixing, in the systems code, the parameters over which we expect to have good control, for example the radial build and toroidal magnetic field. Then the systems code is run many times, sampling the uncertain parameters from distributions which represent the 'known unknowns' about the system performance. Current work focuses mainly on physics parameters but this will be expanded in the future to take account of technological factors such as critical current density in the superconductors and the resulting variation in available field.

Some early results are shown in figure 2. Here the plant parameters of interest are net electrical power output and burn time, as high-level performance targets. The choice of uncertain variables is fully described in reference [11] but is summarised in table I. These results, for initial estimates of the uncertainties, tend to show that the majority of possible outcomes will produce net electrical power in excess of $400 \mathrm{MW}$ and a burn time of more than of $1 \mathrm{hr}$. However, the choice of uncertain parameters and the ranges of 


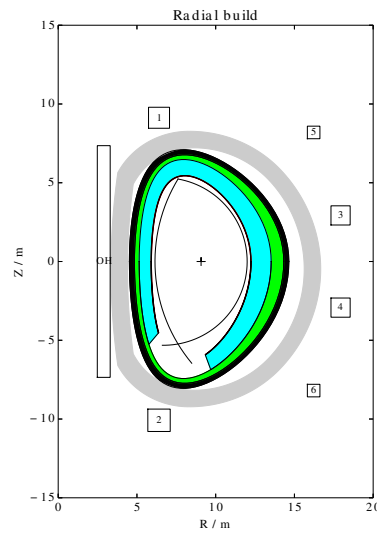

\begin{tabular}{|l|c|}
\hline Parameter & DEMO1 \\
\hline$R_{0}$ & $9.1 \mathrm{~m}$ \\
$A$ & 3.1 \\
$\kappa_{95}$ & 1.59 \\
$P_{\text {fus }}$ & $2 \mathrm{GW}$ \\
$B_{T}$ & $5.7 \mathrm{~T}$ \\
$\beta_{N}$ & 2.6 \\
$H$ & 1.1 \\
\hline
\end{tabular}

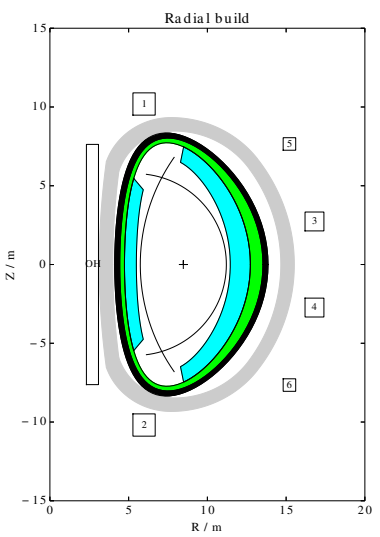

FIG. 1: As the EUROfusion Roadmap is restructured, there is opportunity to reconsider a wider variety of pre-conceptual design options in more detail, leading to a more robust down-selection and greater confidence in the final design [9]. On the left is the cross-section for EU-DEMO, with the major parameters in the centre. On the right is a double-null concept being evaluated.

uncertainty are based on the judgement of the authors in most cases: it would be useful if DEMO-focussed modelling in these areas could help to fix these numbers more accurately.

Single-parameter sensitivity studies have been conducted to explore the effects of varying values around the design point (table II). Here the parameters with the largest impact are $H, \kappa_{95}$, and $\frac{\left\langle n_{e}\right\rangle_{l}}{n_{G}}$. One of these, $\kappa_{95}$, is a design parameter; the other two are functions of the transport physics of the plasma. The strong dependence on $\kappa_{95}$ implies that we must design to operate at the maximum achievable $\kappa$, but this is limited by the fragility of the DEMO first wall: high elongation values may not be achievable due to limitations of the available diagnostics and control systems to stabilise vertical movement. We must have confidence that the scenario is controllable, and further modelling may help here. The uncertainties in $H$ and density limit (above the Greenwald limit here due to profile peaking predicted by transport simulations) can be reduced through careful DEMO-relevant modelling and experiments. This also helps to illustrate the iterative nature of DEMO development: a plant-wide scenario is suggested, and must be evaluated (here in terms of wall loads and controllability), and then the results and recommendations from these calculations are fed back into the scenario development, possibly with implications for many other plant systems.

Work is continuing in this area to assess the inputs which have maximum impact on the output in the multi-dimensional analysis, particularly those which interact to enhance or degrade performance further, and to extend the work to be able to optimise designs accounting for uncertainties to develop the most robust design points with acceptable performance. 


\begin{tabular}{|l|l|c|c|c|}
\hline Parameter & Distribution & Mean & $\sigma$ & DEMO \\
\hline Upper bound on $\frac{\left\langle n_{e}\right\rangle_{l}}{n_{G}}$ & l/h Gaussian & 1.2 & 0.1 & 1.2 \\
Upper bound on H-factor & l/h Gaussian & 1.2 & 0.1 & 1.1 \\
Core radius for radiation correction & Gaussian & 0.6 & 0.15 & 0.6 \\
Thermal $\alpha$-particle fraction & Gaussian & 0.1 & 0.025 & 0.1 \\
$\frac{n_{W}}{n_{e}}$ & Gaussian & $10^{-4}$ & $5 \times 10^{-5}$ & $5 \times 10^{-5}$ \\
Maximum ratio of $\frac{P_{\text {sep }}}{R}(\mathrm{MW} / \mathrm{m})$ & Gaussian & 15 & 2 & 17 \\
Lower bound on L-H threshold limit & Gaussian & 1.0 & 0.25 & 1.0 \\
Bootstrap current fraction multiplier & Gaussian & 1.0 & 0.1 & 1.0 \\
\hline
\end{tabular}

TABLE I: PARAMETERS AND THEIR VARIATION FOR THE UNCERTAINTIES STUDY [1]. UNCERTAINTY DISTRIBUTIONS ARE MAINLY EDUCATED GUESSES, WITH THE EXCEPTION OF THE L-H THRESHOLD POWER FOR WHICH CONFIDENCE LIMITS ARE GIVEN IN [12]. ('L/H Gaussian' indicates 'LOWER halF Gaussian'. $\left\langle n_{e}\right\rangle_{l}$ IS The LineAVERAGED ELECTRON DENSITY, $n_{G}$ THE GREENWALD DENSITY LIMIT, $n_{W}$ THE DENSITY OF TUNGSTEN)

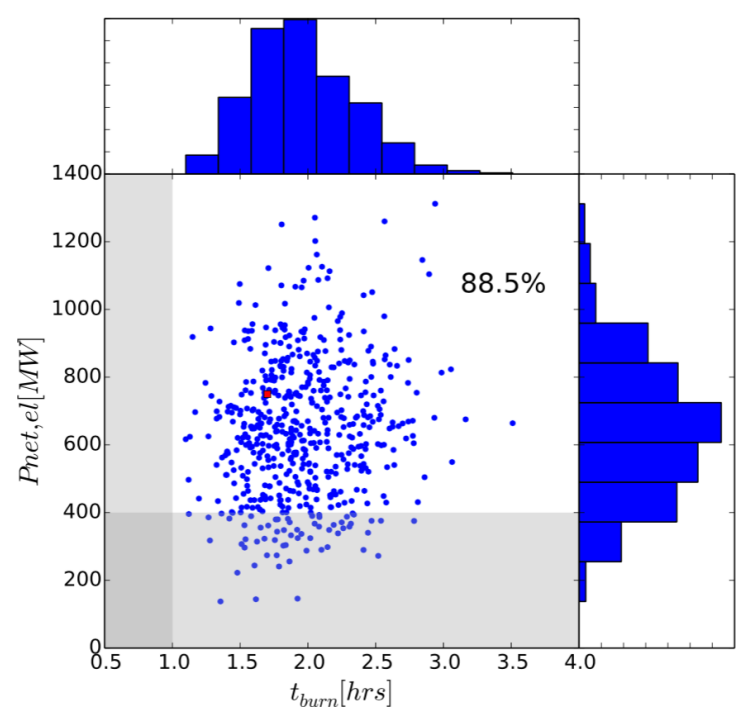

FIG. 2: Predicted performance of a plant built to the EU-DEMO operating point for uncertain physics parameters. Fusion gain $Q$ has been optimised. Nearly $90 \%$ of the points have acceptable output and burn time. As, in a real plant, the balance of plant could not operate much above design capacity there would be scope to trade off excess fusion power for additional burn time in the 'overperforming' cases [11]. 


\begin{tabular}{|l|c|c|c|c|}
\cline { 2 - 5 } \multicolumn{1}{c|}{} & \multicolumn{2}{c|}{$P_{\mathrm{e}, \text { net }}$} & \multicolumn{2}{c|}{$\tau_{\text {pulse }}$} \\
\cline { 2 - 5 } \multicolumn{1}{c|}{} & $-10 \%$ & $+10 \%$ & $-10 \%$ & $+10 \%$ \\
\hline$H$ & $-35 \%$ & $27 \%$ & $-8 \%$ & $15 \%$ \\
$\kappa_{95}$ & $-75 \%$ & $125 \%$ & $28 \%$ & $-7 \%$ \\
$\delta_{95}$ & $-12 \%$ & $13 \%$ & $3 \%$ & $-3 \%$ \\
$c_{\mathrm{W}}$ & $0 \%$ & $0 \%$ & $1 \%$ & $17 \%$ \\
$c_{\mathrm{He}}$ & $10 \%$ & $-9 \%$ & $4 \%$ & $29 \%$ \\
$P_{\mathrm{aux}}$ & $1 \%$ & $-1 \%$ & $-1 \%$ & $1 \%$ \\
$\frac{P_{\mathrm{sep}}}{R}$ & $-3 \%$ & $3 \%$ & $-5 \%$ & $5 \%$ \\
$\eta_{\mathrm{WP}, \mathrm{CD}}$ & $-3 \%$ & $2 \%$ & $0 \%$ & $0 \%$ \\
$\frac{\left\langle n_{e}\right\rangle_{l}}{n_{G}}$ & $-28 \%$ & $30 \%$ & $5 \%$ & $-2 \%$ \\
$\frac{T_{0}}{\left\langle T_{e}\right\rangle}$ & $-2 \%$ & $1 \%$ & $-1 \%$ & $1 \%$ \\
\hline
\end{tabular}

TABLE II: RELATIVE IMPACT OF A MODIFICATION OF $\pm 10 \%$ OF A NUMBER OF INPUT PARAMETERS ON THE NET ELECTRIC POWER AND PULSE DURATION: ALL PROCESS RUNS HAVE BEEN CARRIED OUT WITH FIXED MAJOR RADIUS AND OPTIMISING THE RATIO FUSION POWER AND INJECTION POWER [7]. $c_{\mathrm{W}}$ AND $c_{\mathrm{He}}$ ARE THE FRACTIONAL CONCENTRATIONS OF TUNGSTEN AND HELIUM ASH RESPECTIVELY.

\section{Targeting robust designs}

It is also important to carry out systematic sensitivity studies to help further ascertain which parameters have the largest impact on plant design and performance. A power plant design seldom leans on all performance limits simultaneously and assuming that it does can lead to confounding results (for example, see [13]). Thus understanding which limits (engineering and physics) are being pushed enables research effort to be focused where it will have the greatest effect on machine design. An example is shown in figure 3 . This figure shows the interaction of divertor load limits (represented as $\frac{P_{\mathrm{sep}}}{R_{0}}$ ) and quality of $\mathrm{H}$-mode, represented by the power across the separatrix divided by the H-mode threshold power $f_{\mathrm{LH}}=\frac{P_{\mathrm{sep}}}{P_{\mathrm{LH}}}[12$. Increasing the divertor power limit allows a better H-mode to be obtained and a marginally smaller machine to be designed for a given magnetic field limit (here applied at the coils, and a function of superconductor performance and material stress limits). However, attempting to improve the H-mode at a given divertor power limit forces the solution towards a lower $B_{T}$ (for a fixed $\frac{P_{\text {sep }}}{R_{0}}$, applying the Martin scaling gives $f_{\mathrm{LH}} \sim B^{-1.5}$ ) and the machine size must increase to recover target performance.

An additional complication arises from considering the forces on the TF coils, for which performance is limited not just by the superconductor but also the strength of the structural materials. Further work has shown that dramatic improvements in superconductor performance (for example, using high-temperature superconductors) are soon rendered ineffective unless much stronger materials can also be identified. 


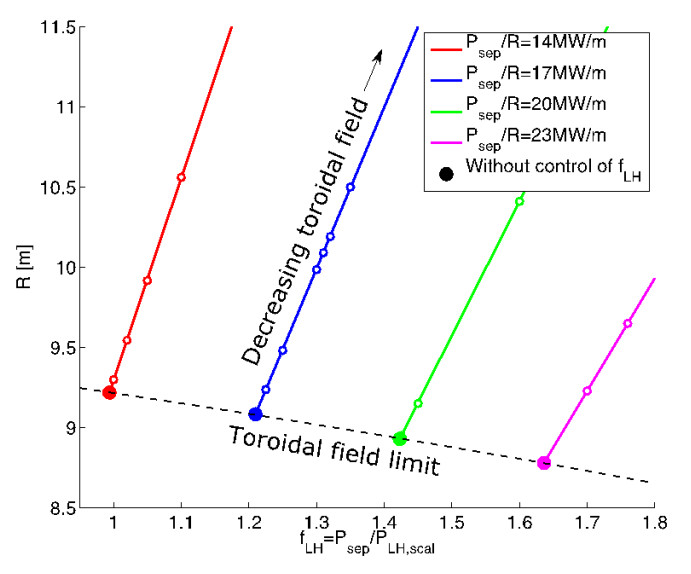

FIG. 3: Dependence of the major radius $R_{0}$ on $f_{\mathrm{LH}}$ for different values of $\frac{P_{\mathrm{sep}}}{R_{0}}$ and respecting the limits of $\mathrm{Nb}_{3} \mathrm{Sn}$ superconductor and magnet structural steels (based on ITER magnets) [14]. The uncertainty in the $L-H$ threshold power is around $50 \%$ for DEMO scenarios [12]. Improved divertor capability therefore provides a mitigation against H-mode being harder to access than expected.

\section{Creating a DEMO 'conceptual risk' register}

One further reason that it is important to gain a good handle on how uncertainties affect plant performance and hence design margins is to consider the steps from ITER to a power plant. Currently EU DEMO assumes relatively low performance (compared to many other international demonstration plant designs) in order to maximise the chances of successful operation. This approach is driven by current physics knowledge and technology limitations, and results in a large and thus expensive system, particularly making the engineering of remote handling and magnet manufacture more difficult. However, working back from an attractive power plant to consider the target performance of DEMO and ITER [15] results in a design point for DEMO which requires advanced plasma performance and has very little margin for underperformance. The conceptual design process must iterate from both directions to find a DEMO which is technologically achievable, has robust performance scenarios, and offers a convincing step towards a power plant. To identify this design point we must minimise the effects of uncertainties on the final system through careful design and experiments which reduce the uncertainties in key areas.

The performance uncertainties described here, and their knock-on effects on plant design and performance, are summarised in a 'conceptual risk' register. This contains entries for the main areas of uncertainty and unresolved problems in future physics and system performance for DEMO; summaries of the consequences on overall plant output if unresolved before the plant design is finalised, or effects on the design to recover output if they are considered unresolvable; and possible mitigation routes. The purpose of this document is to make explicit the design choices and areas where improved modelling and DEMO-relevant experiments will have the greatest impact on confidence in a successful 
DEMO design. As risks are closed out through improved modelling, design, and experimental data, it will also act as a record of why some fundamental design choices were made. One example entry covers the risk posed by unmitigated disruptions, in which the wall load may reach twice the $\mathrm{W}$ melt threshold [16]. A potential solution is to fit mitigation systems at the cost of additional vessel penetrations and loss of tritiumbreeding wall area. An alternative solution could be the identification of well-understood and -controlled scenarios which are far from disruptivity, but whose lower fusion power density would require changes to the radial build of the machine. In order to properly understand these risks, a programme of DEMO-relevant experiments aimed at developing low-disruptivity scenarios with significantly-limited diagnostic and control capabilities (compared to existing experimental devices) needs to be instituted.

One further example is the coupling of the scrape-off layer power to the divertor surface, the solution to which is one of the EUROfusion Missions [17]. If we must assume that DEMO operates in H-mode, there is a lower limit on the input power [12] as well as an upper limit on the power which can reach the divertor plate. As discussed in section 3, the way in which these two limits couple can have significant effects on machine design. The usual solution is to assume most of the power can be radiated away before it is conducted to the plate. Efforts in reducing the uncertainties in this area, from modelling activities and experiments on highly-radiating plasmas and alternative magnetic configurations, would be very valuable in building confidence in DEMO design.

\section{Conclusions}

In order to have confidence that (a) the design of DEMO is a suitable step to a power plant and (b) DEMO will effectively demonstrate target performance, we require both a reasonable image of a future power plant extrapolated from current experimental and projected DEMO results and an assessment of how uncertainties in current models extrapolate through these systems. We must design-in sufficient performance margin that the expected likely actual performance range allows us to achieve targets. This work provides a framework for evaluating the effectiveness of the performance margins, and outlines steps being taken towards making the DEMO design much more robust and allowing us to target research where it has the most effect in increasing confidence in DEMO, allowing us to reduce margins and potentially make DEMO cheaper. This work is intended to make explicit the design choices and areas where improved modelling and DEMO-relevant experiments will have the greatest impact on confidence in a successful DEMO design.

\section{Acknowledgements}

This work has been carried out within the framework of the EUROfusion Consortium and has received funding from the Euratom research and training programme 2014-2018 under grant agreement No 633053 and from the RCUK Energy Programme [grant number EP/I501045]. The views and opinions expressed herein do not necessarily reflect those of the European Commission. 


\section{References}

[1] P. Batistoni, et al. Report of the ad hoc group on DEMO activities. Technical Report CCE-FU 49/6.7, IPP, Garching, March 2010.

[2] G. Federici, et al. Overview of EU DEMO design and R\&D activities. Fusion Engineering and Design, 89:882-889, 2014.

[3] M. Kovari, et al. "process": A systems code for fusion power plants - part 1: Physics. Fusion Engineering and Design, 89(12):3054-3069, 2014.

[4] M. Kovari, et al. "process": A systems code for fusion power plants - part 2: Engineering. Fusion Engineering and Design, 104:9-20, 2016.

[5] D. Maisonnier, et al. The European power plant conceptual study. Fusion Engineering and Design, 75-79:1173-1179, 2005.

[6] R. Wenninger, et al. Advances in the physics basis for the european DEMO design. In 25th Fusion Energy Conference. IAEA, St Petersburg, October 2014.

[7] R. Wenninger et al. The physics and technology basis entering european system code studies for DEMO. Accepted by Nuclear Fusion, 2016.

[8] M. Coleman, et al. On the $\{$ EU $\}$ approach for $\{$ DEMO $\}$ architecture exploration and dealing with uncertainties. Fusion Engineering and Design, 109-111, Part B:1158 - 1162, 2016. Proceedings of the 12th International Symposium on Fusion Nuclear Technology-12 (ISFNT-12).

[9] M. Coleman. The implementation strategy for the pre-conceptual and conceptual design of a european demo. In 22nd Topical Meeting on the Technology of Fusion Energy (TOFE). Philadelphia, USA, August 2016.

[10] https://www.euro-fusion.org/newsletter/fusion-roadmap/.

[11] H. Lux, et al. Uncertainties in power plant design point evaluations. In 29th Symposium on Fusion Technology (SOFT). Prague, September 2016.

[12] Y. R. Martin, et al. Power requirement for accessing the H-mode in ITER. Journal of Physics: Conference Series, 123:012033, 2008.

[13] J. P. Freidberg, et al. Designing a tokamak fusion reactor - how does plasma physics fit in? Physics of Plasmas, 22:070901, 2015.

[14] R. Wenninger, et al. Demo exhaust challenges beyond iter. In 42nd EPS Conference on Plasma Physics, page P4.110. Lisbon, June 2015.

[15] H Zohm, et al. Where to locate demo in a one-step-to-an-fpp strategy. In 43rd EPS Conference on Plasma Physics, page P4.077. Leuven, July 2016.

[16] R. Wenninger et al. The DEMO wall load challenge. Submitted to Nuclear Fusion, 2016.

[17] M. Turnyanskiy, et al. European roadmap to the realization of fusion energy: Mission for solution on heat-exhaust systems. Fusion Engineering and Design, 96-97:361 - 364, 2015. Proceedings of the 28th Symposium On Fusion Technology (SOFT-28). 